\title{
Menschen kurz vor ihrem Tode besser begleiten
}

\author{
Viele Hausärzte wissen nicht, was sich Patienten kurz vor ihrem Tode wünschen und \\ wie und wo sie sterben möchten. Eine offene, einfühlsame Kommunikation und \\ vorausschauendes Planen kann die Lebensqualität von Sterbenden erhöhen und ihr \\ Leben sogar verlängern. Auf der Swiss Family Docs Konferenz stellten Hausärzte ein \\ spannendes Nationales Forschungsprogramm vor und berichteten, wie man Patien- \\ ten am Lebensende mitfühlend begleitet.
}

Felicitas Witte

Medizinjournalistin
Lebhaft und aktiv war die Dame immer schon gewesen. Sie leidet zwar unter einer Polyarthrose, und ihre Gelenke zwicken hie und da, aber das hindert sie nicht daran, sich liebevoll und aufopfernd um die alten Menschen in ihrem Quartier zu kümmern. Anderen Menschen zu helfen, macht die Frau glücklich und zufrieden. Sie selbst will aber niemandem zur Last fallen. Wenn sie alte Menschen sieht, die von

\section{«Viele Hausärzte wissen nicht, was sich Patienten für ihr}

\section{Lebensende wünschen.»}

* http://de.swissfamilydocs.ch/ 2013/

Korrespondenz: Dr. med. Felicitas Witte Pfeffingerstrasse 37 CH-4053 Basel Tel. 0793776193

felicitas.witte[at]web.de www.felicitas-witte.de ihrer Umgebung nichts mehr mitbekommen, ist ihr einziger Wunsch: So möchte sie nicht enden, sie möchte möglichst lange geistig präsent sein. Doch es kommt alles anders. Kurze Zeit später erleidet sie einen Hirnschlag. Die Hälfte ihres Körpers kann sie nicht mehr bewegen, sie kann nicht mehr sprechen und nicht mehr schlucken. Im Spital legt ihr der Arzt eine Ernährungssonde. Eigentlich hätte die Frau das nie gewollt. Aber sie hat das nie in einer Patientenverfügung geschrieben und ihren Hausarzt oder die Familie darüber informiert. Die Frau wird zum schweren Pflegefall - fünf Jahre lang, bis sie stirbt.

«Viele Hausärzte wissen nicht, was sich Patienten für ihr Lebensende wünschen», berichteten kürzlich Klaus Bally und Hans-Ruedi Banderet, niedergelassene Allgemeinmediziner in Basel auf der Swiss Family Docs Konferenz in Bern*. Sterbende Patienten begleiten die beiden Hausärzte seit Jahrzehnten, doch 2010 begannen sie, sich auch theoretisch mit dem anstaltung, bei der es um die Ausschreibung eines neuen Nationalen Forschungsprogramms (NFP) zum Thema «Lebensende» ging. Im NFP 67 sollte «für Politik und Gesellschaft das einschlägige Wissen erarbeitet werden, damit die letzte Lebensphase alter wie junger Menschen humaner gestaltet werden kann». «Wir bemerkten, dass ausser uns keine Hausärzte da Thema auseinanderzusetzen. Sie waren bei einer Ver- waren», erzählte Bally in Bern. «Aber bei so einem Thema wäre unserer Input doch extrem wichtig.»

\section{Hausärzte forschen im Nationalen Forschungsprogramm}

So bewarben sich die beiden Mediziner, die zusätzlich am Institut für Hausarztmedizin forschen, mit einem Hausarzt-Projekt. «Normalerweise werden Hausärzte nicht gerade überflutet mit NFP-Projekten», sagte Bally. «Zu unserem Erstaunen wurde unser HausarztProjekt angenommen.» Dem NFP stehen insgesamt 15 Millionen Franken zur Verfügung, es soll bis 2018 dauern. In ihrem Teilprojekt untersuchen Bally und Banderet gemeinsam mit anderen Forschern, wie gut Patienten am Lebensende betreut sind - und zwar einmal aus Sicht der Hausärzte und einmal aus Sicht der Angehörigen. «An unserem Projekt arbeiten Wissenschafter aus verschiedenen Bereichen, etwa Medizinethik, Palliativmedizin, Allgemeinmedizin, Psychologie oder Epidemiologie», so Bally. «So können wir die unterschiedlichen Aspekte am besten beurteilen.» Mit Interviews und Fragebögen in allen drei Sprachregionen der Schweiz soll die Qualität der Gesundheitsversorgung erfasst und mit den Erkenntnissen sollen Lücken in der Betreuung und vor allem in der hausärztlichen Versorgung von Menschen am Lebensende erkannt und verbessert werden. «Das soll

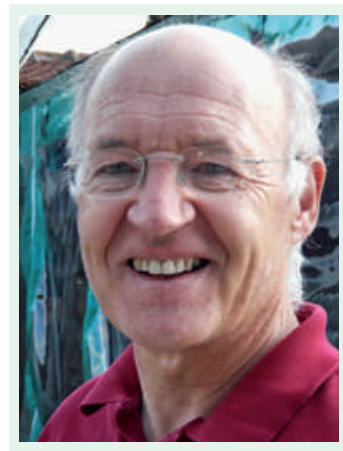

Hans-Ruedi Banderet: «Patient, Familie und Hausarzt sollten wissen, was der Patient wünscht.»

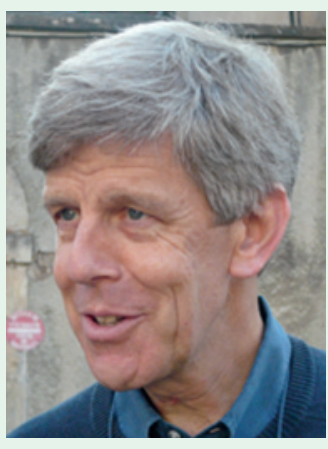

Klaus Bally: «Normalerweise werden Hausärzte nicht gerade überflutet mit NFP-Projekten.» 
die Lebensqualität der Menschen kurz vor ihrem Tode verbessern», hofft Bally.

Und die scheint nicht so gut zu sein, wie man sich das wünschen könnte. Gemäss einer Studie aus den Niederlanden kennen nur 46 Prozent der Hausärzte den bevorzugten Sterbeort ihrer Patienten [1]. Das, was Ärzte für die Bedürfnisse ihrer Patienten halten, stimmt gemäss einer Studie aus den USA oft nicht mit dem überein,was die Patienten wünschen [2]. So war es beispielsweise 89 Prozent der Patienten wichtig, der Familie nicht zur Last zu fallen. Dass sich das Patienten wünschen, vermuteten aber nur 58 Prozent der Ärzte. 92 Prozent der Patienten hofften, am Ende ihres Lebens mental bei vollem Bewusstsein zu sein, aber nur 65 Prozent der Ärzte hielten das für wichtig. «Wir vermuten, dass es solche Unterschiede in der Schweiz auch gibt», sagte Bally. «Mit unserer Studie werden wir genau wissen, welche.» Um die Wünsche von Patienten kurz vor ihrem Tode besser herauszufinden, stellte der Mediziner ein Kartenspiel vor, das «Go-Wish-Spiel». Auf Spielkarten ist dabei jeweils ein Wunsch geschrieben, den ein Patient am Lebensende haben könnte. Also zum Beispiel keine Schmerzen zu spüren, im Frieden mit Gott zu sein, sich von seinen Lieben in Ruhe zu verabschieden oder eine Pflegerin zu haben, mit der man sich wohl fühlt. Der Patient zieht eine Karte und soll dann bei jeder Aussage angeben, ob dieser Wunsch für ihn «sehr wichtig», «etwas wichtig» oder «nicht wichtig» sei. «In spielerischer Atmosphäre kann man so herausfinden, was dem Patienten wichtig ist, ohne ihn mit Fragen zu sehr zu drängen», sagt Bally. Man solle sich trauen, die Patienten auf ihre Wünsche anzusprechen, und das Lebensende planen. Man könnte den Patienten etwa fragen, ob man ihn vorsorglich im Hospiz anmelden solle, was er möchte, wenn es zu Hause nicht mehr weitergehe oder ob man gemeinsam mit der Familie sprechen solle. «Palliativmedizin ist eine hausärztliche Aufgabe», sagte Bally. «Die Patienten sind in der Regel dankbar, wenn wir sie ansprechen und mitfühlend begleiten.» Das kann nicht nur die Lebensqualität verbessern, sondern auch lebensverlängernd wirken, wie eine Studie bei Lungenkrebspatienten ergab [3].

\section{Ein Betreuungsnetz für das Lebensende}

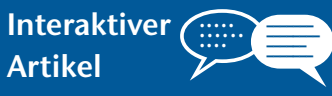

Wollen Sie diesen Artikel kommentieren? Nutzen Sie dafür die Kommentarfunktion in der OnlineVersion oder sehen Sie nach, was Ihre Kolleginnen und Kollegen bereits geschrieben haben: www.saez.ch/ aktuelle-ausgabe/ interaktive-beitraege/

«Man sollte zusammen eine gemeinsame Richtung definieren», sagte Banderet. «Patient, Familie und Hausarzt sollten wissen, was der Patient wünscht und wie er sein Leben kurz vor dem Tode verbringen möchte.» Auch wenn es sich mehr wie ein BusinessPlan anhört als die Vorbereitung auf den Tod: Alles sollte gut geplant, besprochen und mit verständlichen Worten schriftlich festgelegt werden. Dazu ge-
Patient sterben möchte, ob er lebenserhaltende Massnahmen wünscht oder eine terminale Sedation in Frage kommt. «Man sollte das Thema rechtzeitig ansprechen, nicht erst kurz vor dem Tod, wenn der Patient kaum noch Kraft hat», riet Banderet.

Schritt für Schritt solle man vorgehen und den Patienten nicht mit zu vielen Planungen bedrängen. «Es ist auch völlig in Ordnung, wenn der Patient sich zu dem Zeitpunkt noch nicht entscheiden kann solche Ambivalenz ist völlig normal», sagte Banderet. Der Hausarzt ist dann der Koordinator des Netzwerkes aus Patient, Familienmitgliedern, Freunden, Spitalärzten, Pflegenden und anderen Beteiligten. Er übernimmt die Verantwortung und sorgt dafür, dass alle stets informiert sind. So ein Netz könne man als «Hospitalisation zu Hause» betrachten, erklärte Banderet. Wenn man sieht, dass das Netz einzureissen droht, zum Beispiel weil es dem Patienten kurz vor dem Wochenende plötzlich schlechter geht, könnte man proaktiv ein Hospiz informieren und ankündigen, dass der Patient möglicherweise kurzfristig kommt. «Notfälle muss man vorausschauend planen», riet Banderet. «Und ausserdem genügend Reservemedikation verschreiben und sicherstellen, dass immer ein Ansprechpartner erreichbar ist.»

Die Betreuung und das Netz enden nicht mit dem Tod. «Am besten trifft man sich mit den Mitgliedern des Betreuungsnetzes nach dem Tod des Patienten und redet über die Zeit», riet Banderet. Verwandte hätten zum Beispiel häufig Schuldgefühle, dass sie etwas falsch gemacht hätten. «Dabei ist Begleitung am Lebensende gerade für nichtprofessionelle Helfer eine enorme Belastung.» Einen Menschen bis zum Tode zu betreuen, sei oft eine Vorbereitung auf den eigenen Tod, erzählte Banderet. So berichtete er von einem 77-jährigen Mann mit unheilbarem Prostatakarzinom, den er eng gemeinsam mit der Familie bis zu dessen Tode begleitete. «Das löst viele Emotionen bei einem selbst aus, die man dann erst einmal verarbeiten muss.»

\section{Literatur}

1 Meeussen K et al. GPs' awareness of patients' preference for place of death. Br J Gen Pract. 2009;59:665-70.

2 Steinhauser KE et al. Factors considered important at the end of life by patients, family, physicians, and other care providers. JAMA. 2000;284:2476-82.

3 Temel JS et al. Early Palliative Care for Patients with Metastatic Non-Small-Cell Lung Cancer. NEJM. 2010; 363:733-42.

\section{Weiterführende Informationen}

- www.nfp67.ch hört, den Pflege- und Sterbeort festzulegen, wie der
- www.snf.ch/D/Aktuell/SNFInfo/NFP-NFS/Seiten/ default.aspx?NEWSID=1794\&WEBID=705D0BF9 BC95-43E6-BF65-F8B316A4D74E

- www.nfp67.ch/D/projekte/sterbeverlaeufe-undversorgung/betreuungsqualitaet-am-lebensende-sichthausaerzte-angehoerige/Seiten/default.aspx

- www.primary-care.ch/docs/primarycare/2013/06/de/ pc-d-00286.pdf 